\title{
Function of BMPs and BMP Antagonists in Adult Bone
}

\author{
ETSUKO ABE \\ Mount Sinai Bone Program, Department of Medicine, Mount Sinai School \\ of Medicine, New York, NY 10029 and Bronx VA Medical Center, Bronx, \\ NY 10468, USA
}

\begin{abstract}
The expression and function of BMPs and BMPs in bone tissues have been studied for a long time because of their remarkable activities. However, their biological functions in normal bone remodeling in adults were not fully understood until recently. Advanced technologies using gene manipulation were used to study their roles in adulthood. In addition, findings of new BMP antagonists and the effect of Wntcanonical pathways on bone features also provided new insights in bone studies.
\end{abstract}

KEYWORDS: BMP; BMP antagonist; Wnt; catenin; osteoblast; bone remodeling

\section{INTRODUCTION}

Bone morphogenetic proteins (BMPs) belong to the transforming growth factor (TGF)- $\beta$ superfamily, which includes TGF- $\beta$, activin, and inhibin. ${ }^{1}$ BMP receptors are present in a variety of tissues and cells, and thus BMPs display numerous functions that are not limited in mesodermal development. Recent studies demonstrated new roles of BMPs in brain, heart, kidney, skin, and intestine. ${ }^{2,3}$ In bone tissues comprising two distinct cell types, chondrocytes and osteoblasts, BMPs and their antagonists are temporally and spatially expressed, and both factors are necessary for normal development. ${ }^{4-6}$ Thus, if the functions are lost or gained, severe abnormalities are observed and thereby cause death in embryonal stage or in postnatal stage. In bone cells using ex vivo system, BMPs and BMP antagonists endogenously express and regulate cell differentiation. ${ }^{7,8}$ The addition of those factors accelerates or inhibits cell differentiation in cartilage and bone. Based on the results we have hypothesized that BMPs and BMP antagonists are required for normal bone remodeling in adulthood as well as embryonic development; however, the information

Address for correspondence: Etsuko Abe, Division of Endocrinology, Department of Medicine, Mount Sinai School of Medicine, One Gustave L Levy Place, Box 1055, New York, NY 10029. Voice: 212-241-8735; fax: 212-241-4218.

e-mail: etsuko.abe@mssm.edu

Ann. N.Y. Acad. Sci. 1068: 41-53 (2006). (C) 2006 New York Academy of Sciences.

doi: 10.1196/annals.1346.007 
to prove this hypothesis was limited. Current technologies using conditional knockout mice or transgenic mice with inducible system gave new insight into BMPs and BMP antagonists on bone remodeling. Furthermore, the new signals of Wingless type (Wnt)-canonical pathway ${ }^{9}$ in addition to BMP signaling ${ }^{10-12}$ provide a new strategy to understand bone biology under normal and pathological conditions.

\section{BMP AND BMP SIGNALING}

BMPs bind to BMP receptors types I and II, both of which are required for signal transduction. ${ }^{10-12}$ There are seven distinct BMP type I receptors, including ALK-2, ALK-3 (BMPRIA), and ALK-6 (BMPRIB), and BMP type I receptor mainly determines the specificity of the intracellular signals. BMP type II also has distinct receptors, such as BMP type II receptor (BMPRII), activin type II receptor (ActR-II), and activin type IIB receptor (ActR-IIB). ALK-3 ubiquitously expressed in most types of cells including osteoblasts. Chondrocytes and osteoclasts are known to express only ALK-6. A common feature of the TGF- $\beta$ superfamily has seven conserved cysteins. BMPs have two extra-domains in addition to the structure identified by another TGF family, TGF- $\beta$, activin/inhibin, or nodal. BMPs are translated as a large molecule with a signal peptide and cleaved to be a dimeric form of mature protein with $25 \mathrm{kD}$ after secretion. Currently, over 20 distinct BMPs have been identified and each of them has slightly different affinities to their receptors. BMP-2, -4, -6, and -7 (OP-1) are widely accepted to have osteoinductive activities in vivo. ${ }^{1}$ Other BMPs, ${ }^{12}$ namely GDF (growth differentiation factor)-5, -6, and -7 (BMP12) and activin/inhibin family, exhibit low osteoinductive activity by themselves, but modulate BMP action. Both BMP-2 and -4 preferentially bind to ALK-3 and -6 and transduce signals through smad-dependent and/or independent mechanisms.

After binding to their receptors, BMPs activate intracellular transcription factors, smad- $1,-5$ and -8 (smad-1/5/8, R-smads) and proceed toward dimerization with smad-4 (Co-smad, common smad) before translocation into nucleus. ${ }^{10-12}$ The generated complexes are then translocated in the nucleus to regulate specific gene transcription (FIG. 1). Osteopontin, osteoprotegerin, BMP-7, and smad-1 are known to be activated through The BMP-induced smad activation mechanism. ${ }^{13-15}$ Similar to the BMPs, TGF- $\beta$ and activin stimulate smad-2 and -3 (smad-2/3, R-smads) activation by generating complex with smad-4. Importantly, inhibitory smads, smad-6 and -7 (I-smads) exist to negatively regulate TGF- $\beta$ superfamily signaling; they do so by binding to the BMP/TGF- $\beta$ receptors or binding to the smad- 1 and thereby intefering with activation.

Smad-independent signals are also required for the maximum activation of BMP signals in osteoblasts and chondrocytes. BMPs stimulate MAP 


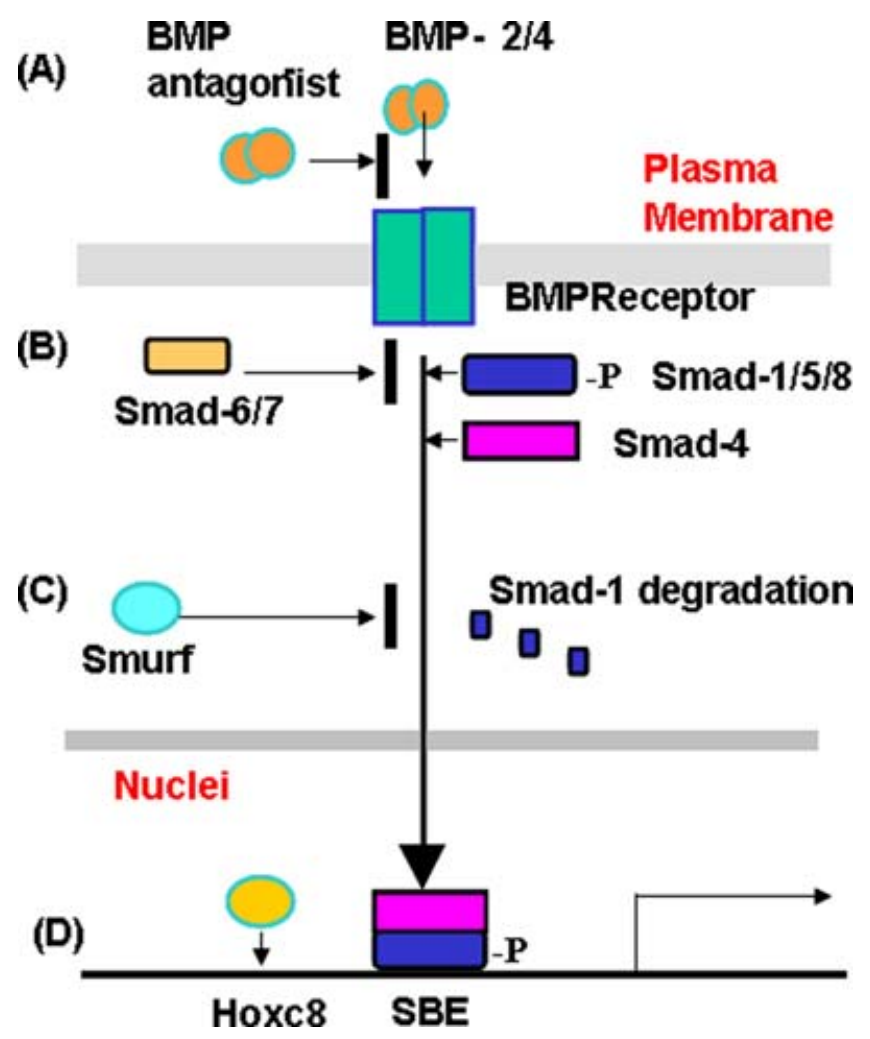

FIGURE 1. BMP signaling and its inhibition. There are four distinct inhibitions for BMP signaling. (A) BMP antagonists inhibit binding of BMP-2/4 to their receptors. (B) Smad-6/7 completes binding to the BMP receptors with smad-1/5/8 for the inhibition of BMP signaling. (C) Smurf 2 promotes smad-1 degradation after ubiquitination. (D) Transcription factor (Hoxc8) binds to target genes to inhibit BMP-induced transcription.

kinase, pI3 kinase, and/or JNK (c-Jun N-terminal kinase) ${ }^{16,17}$ and application of the specific inhibitors for these signals diminish expression of osteoblast and chondrocyte differentiation markers, supporting the important role of smadindependent mechanism in BMP signaling as well.

\section{BMP ANTAGONISTS}

BMP antagonists were found in the Spemann organizer of Xenopus embryos as a molecule to inhibit BMP binding to their receptors. They are designated as noggin and chordin. ${ }^{18,19}$ After the first discovery of the BMP antagonists, numerous BMP antagonists, which are secretory proteins with cysteine arrangement structure, were found. ${ }^{20,21}$ They are now classified into three subfamilies based on the size of the cysteine knot: the DAN family, twisted gastrulation, 
and noggin/chordin. Dan family is further subdivided to four groups: Gremlin/PRDC, Cerberus/Cer 1, Dan, and sclerostin/USAG-1. Tsukushi (TSK), which recently was found as a new family of the BMP antagonist, cooperates with other BMP antagonists. ${ }^{22}$

Noggin binds to BMP-2 and -4 with high affinity and to BMP-6 and -7 with low affinity to prevent further action of BMP action. Null mice of noggindisplayed impaired joint formation and thinning calvarial formation with small numbers of osteogenic progenitors thereby die just after birth. ${ }^{23}$ Heterozygous show normal growth and normal bone formation in mice; however, in human apical joint fusion has appeared, ${ }^{24}$ indicating that functional incompletion occurred in the haplotype of the gene. Noggin expression appears in the early stage of embryonal development: node, notochord, dorsal somite, condensing cartilage, and immature chondrocyte are positive for noggin..$^{23}$ In adult mice, high expression of noggin expression was observed in the limited tissues and cells, such as osteoblasts, chondrocytes, and cranial suture in vertebrae and calvaria. ${ }^{25,26}$ Interestingly, the expression of noggin as well as BMPs and the BMP receptor (ALK-3) in macrophages serve to regulate osteoclast formation. ${ }^{26}$ In pathological conditions, such as bone fracture healing, BMPs as well as noggin are intensively expressed at the fracture site ${ }^{27,28}$ and serve to stimulate bone formation.

Noggin transgenic mice are generated by two groups using a rat or mouse osteocalcin promotor. ${ }^{26,29}$ The use of rat osteocalcin promotor $(1.7 \mathrm{~kb})$ displays severely impaired bone formation with bone fracture at young age (4-5 weeks) ${ }^{29}$ In contrast, the use of mouse osteocalcin promotor $(1.3 \mathrm{~kb})$ displays effectiveness in bone remodeling in adult animals. ${ }^{26}$ Bone mineral density was reduced at 6 months old and bone formation rate was significantly suppressed at 8 months old with diminished osteoblast differentiation markers. Notably, osteoclast progenitor numbers are also decreased by noggin overexpression in osteoblasts, indicating that impaired osteoblast function affects osteoclast formation through diminished RANKL (receptor activator of NF- $\mathrm{kB}$ ) expression in osteoblasts.

Chordin as well as noggin bind BMPs and modulate BMP action. ${ }^{30,31}$ The biological activity of chordin resides in the cysteine-rich domains (CRs), specifically in CR1 and CR3 regions, and individual CRs reveal lower activity than that of full-length chordin. As a result, the cleavage of the chordin by an extracellular zinc metalloproteinase, Tolloid/BMP-1, would release a molecule with lower anti-BMP activity than an intact one. Both noggin and chordin display similar BMP antagonistic activity; however, both factors are required for normal head and facial development, ${ }^{32}$ since their expression patterns are different from each other in terms of location and timing. The chordin appears in an earlier stage of embryo than noggin. In the mid-gastrula stage, the expression of noggin and chordin patterns overlap, indicating that they might compensate for each other if one of the two is lost. Conversely, if both chordin and noggin are mutated, mice display severe defectiveness in development. 
Twisted gastrulation (TSG) is expressed in lung, thymus, and kidney,${ }^{20}$ and binds to BMPs to inhibit BMP action in osteoblasts. ${ }^{33}$ This process cooperates with the other BMP antagonist, chordin. TSG null mice show growth retardation after birth, and display dwarfism with delayed endochondral ossification with lymphopenia, ${ }^{34}$ followed by death within a month. The bone phenotypes with decreased bone mineral density were similar to those of the transgenic mice expressing a truncated dominant-negative BMP type 1B receptor (ALK6). Is TSG BMP agonist or antagonist? Interestingly, TSG null mice displayed lymphoid cell depletion with small sizes of spleen and thymus, ${ }^{34}$ suggesting that TSG might act as an agonist. However, smad-1 signals in the TSG null thymocyte were intensively activated without stimulation, ${ }^{34}$ indicating that TSG does act as an antagonist. Thus, the action of TSG may be determined in cell-specific manner. Recent studies using TSG overexpression system in stromal/preosteoblasts supported antagonistic activity of TSG on BMP signaling ${ }^{33}$; the cells inhibited smad activation and thereby suppressed osteoblast differentiation. How does the same molecule act either as an antagonist or as an agonist? The mechanism for agonist activity of TSG is explained by indirect action: TSG promotes cleavage of co-associator chordin to enhance to BMP activity.

Sclerostin is a protein newly discovered BMP antagonist in the patients showing sclerosteosis, an autosomal recessive disorder. ${ }^{35}$ Sclerostin expresses in osteogenic cells including hypertrophic chondrocytes, osteoblasts, and osteocytes, and inhibits action of BMP-6 and $-7,{ }^{36-38}$ and in some cases the action of BMP-2 and -4. Thus, loss of sclerostin increases bone mass seen in sclerosteosis; conversely transgenic mice overexpressing sclerostin exhibit low bone mass with reduced osteoblast activity and bone formation activity. ${ }^{37}$ Importantly, the intense expression of sclerostin in osteocyte emerges as a negative regulator ${ }^{38}$ which controls the fate of osteoblasts on bone surface through canalicurae. The high expression of sclerostin similar to other BMP antagonists, is also observed in the long-term culture or after treatment with BMPs. A mechanism for how sclerostin inhibits BMP action is still controversial. Both smad-dependent and -independent mechanisms ${ }^{38,39}$ are proposed. In the case of a smad-independent mechanism, sclerostin binds to LRP-5 and -6 and inhibits Wnt-canonical signaling pathway and thereby suppresses osteoblast differentiation. ${ }^{39}$ Noggin and sclerostin are capable of forming an inhibitory complex as observed for chordin and TSG.

TSK, which was cloned from a chick lens library, is a new BMP antagonist. ${ }^{22}$ TSK displays a unique protein structure with small leucine-rich proteoglycan (LRP), which is a different type of molecule from other the BMP antagonists described above. The expression of TSK in chick development is overlapped with chordin, and TSK cooperates with chordin, not noggin or follistatin, to inhibit BMP-4 action in vitro and in vivo. Thus, TSK similar to chordin, blocks BMP function. The finding of the new protein, which can cooperate with other BMP antagonists, emphasized the important role 
of BMP antagonists in vitro and in vivo and some redundancy among BMP antagonists.

\section{A BALANCE OF BMP AND BMP ANTAGONIST IN BONE REMODELING}

Endogenous BMP expression appears during cell differentiation from mesenchymal cells to osteoblasts. After differentiation, cells express alkaline phosphatase and osteoblast markers, such as collagen type I, bone sialoprotein (BSP), osterix, and osteocalcin. In the late stage cells deposit minerals stained by Von Kossa or alizarin red. Similar to BMP expression, BMP antagonists also appear and increase their expression levels in the late stage. ${ }^{8}$ The expression levels of the BMP antagonists are transcriptionally regulated by $\mathrm{BMPs}^{7}$ and the balance of BMPs and BMP antagonists determines the rate of cell differentiation. If BMPs or BMP antagonists are exogenously added, cells accelerate or stop further differentiation. Interestingly, mesenchymal cell differentiation is inhibited in aged mice or in aged animal models, which show osteopenia at a young age, namely senescence-accelerated mice (SAM). ${ }^{40,41}$ We thought the decreased bone formation might be explained by the imbalance of BMPs and BMP antagonists. We observed that noggin expression is elevated in bone marrow cell cultures and bone tissues of aged animals. ${ }^{26}$ Notably, no changes are seen in BMP expression.

A deficiency of BMPs, BMPR, or smads severely disrupts normal development and thereby animals die in an early stage of development. Conversely, but more importantly, if BMP action is more enhanced like in noggin deficiency, ${ }^{23}$ cartilage area in long bone is enlarged and endochondral bone formation is not developed. In contrast to the deficient animals of BMP antagonists, overexpression of BMPs and their related proteins sometimes show induction of ectopic ossification. Fibrodysplasia ossificans progressiva (FOP) is a patient exhibiting congenital malformations by progressive heterotopic endochondral ossifications. ${ }^{42}$ Transgenic mice using neuron-specific enolase (NSE) promotor displayed severe postnatal heterotopic ossification, ${ }^{43}$ which closely recapitulated major FOP patients. Generated double transgenic mice with NSE-noggin rescued ectopic ossification, suggesting that only BMP may be required for ectopic bone formation in FOP patients.

Transgenic mice of dominant negative (truncated) form of BMPIA receptor (ALK-3) using colla promotor $(2.3 \mathrm{~kb})$ displayed postnatal osteopenia due to reduced bone formation rate and inhibition of osteoblast differentiation. ${ }^{44}$ Similar bone phenotype has been observed in transgenic mice of smurf (smad ubiquitin regulatory factor) 1 using colIal promotor. ${ }^{45}$ Smurf $1 / 2$ is able to inhibit BMP signaling through the enhancement of ubiquitination of smad-1 and also degradation of BMP type I receptor by cooperating with I-smads. These results suggested the critical role of BMP 
signaling in postnatal stage in addition to embryonic stage. Conditional knockout mice of BMPR1A (ALK-3) in osteoblasts using a cre-loxP system and ostecalcin promotor (OG2) have also demonstrated the crucial role of BMPs specifically in adulthood. ${ }^{46}$ The mutant mice at 6 months of age showed decreased bone mineral density, trabecular bone area, and bone formation rate with irregular calcification. Although the bone volume was increased in the mutant mice, this was explained by reduced bone resorption by osteoclasts, rather than increased bone formation. The mutant mice lost more bone after ovariectomy likely resulting from the decreased osteoblast function, which could not overcome ovariectomy-induced bone resorption. Thus, the bone phenotype obtained BMPR1A is very similar to that in the noggin transgenic mice we generated. Mice transgenic for the transcription factors required for BMP signaling may increase osteoblast differentiation and thereby enhance bone formation. Interestingly, mice overexpressing smad-1C using a doxycycline inducible system show enhanced bone mineral density and bone formation parameters, such as increased osteoid surface, thicker trabecular bone with close proximity, and appearance of cuboidal osteoblasts. ${ }^{47} \mathrm{Smad}-$ 1C, which was discovered as a factor that interacts with Hoxc-8 in osteopontin regulation, mimics smad- 1 activity in osteoblast differentiation. These results reinforced that the transcription factors required for BMP signaling have a significant role in adult bone remodeling as well.

\section{ROLE OF WNT-CANONICAL PATHWAY IN BONE AND A CROSS-TALK WITH BMP SIGNALS}

Recent studies of Wnt-canonical pathway evidenced novel role of new proteins in skeletal development and bone remodeling. Wnts are secreted cysteinerich glycoproteins that influence cell proliferation and survival, therefore Wnt signaling and their roles are implicated not only in development but also in some diseases. ${ }^{9}$ In bone tissues Wnt signaling is designated as a required factor and sometimes a mediator of BMP action during normal bone remodeling. Extracellular Wnt ligands interact with a host of secreted antagonists, secreted frizzled-related protein (sFRP), and Dickkopf (Dkk) family members, preventing the activation process (FIG. 2). Under this condition, $\beta$-catenin is degraded in the cytosol and therefore signals are not transduced. Once Wnt concentration is exceeded, Wnts interact with members of the Frizzled (FZ) family, and LDL (low-density lipoprotein receptor-related proteins 5 and 6) leads to further activation through intracellular proteins. This process protects degradation of $\beta$-catenin and stimulates translocation of $\beta$-catenin to the nucleus where specific gene carrying TCF/LEF ( $\mathrm{T}$ cell factor) should be transactivated. In pathological conditions, such as myloma and osteosarcoma, Wnt signaling is enhanced. ${ }^{48,49}$ Myeloma cell lines and primary myloma cells specifically produce sFRP-2, not sFRP-1, or -3 and thereby impair bone formation in vivo. 


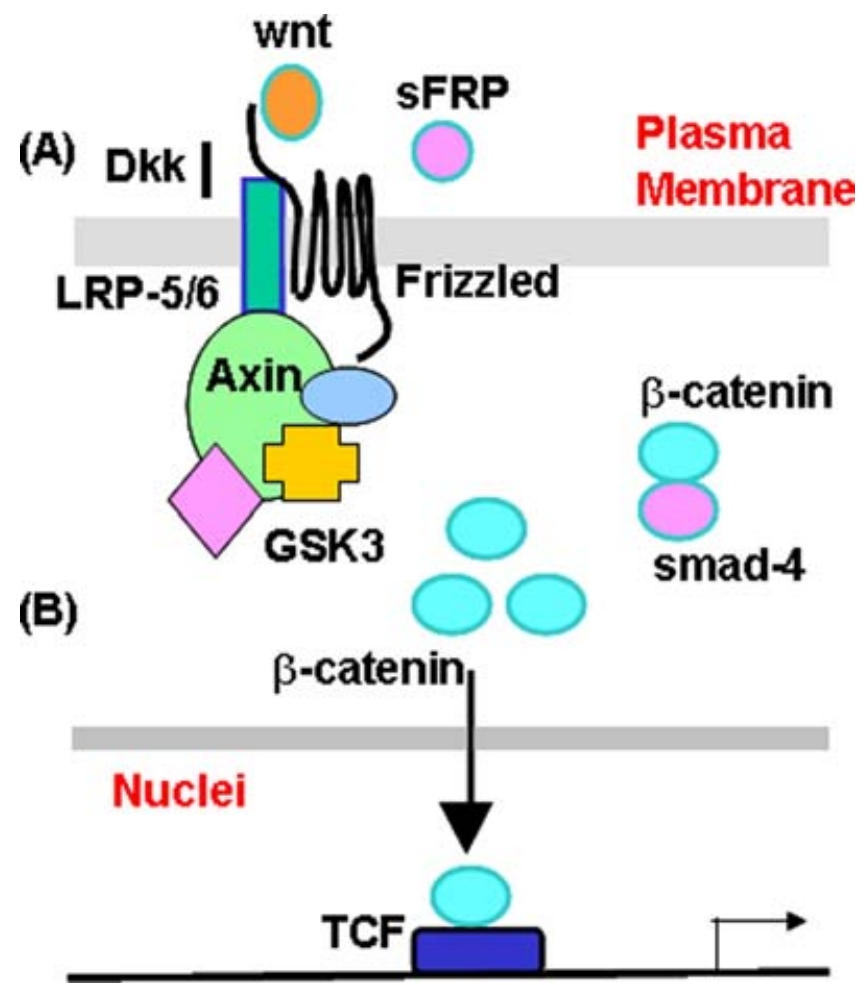

FIGURE 2. Wnt-canonical pathway and cooperation with BMP signaling. Wnt binds to the receptor (Frizzled) with co-receptor (LRP-5/6) and activates downstream signals with GSK3 (glycogen synthase kinase) complex. Under an activation condition, $\beta$-catenin is in stable form, translocated into the nucleus to bind to the TCF sequence in target gene. DKK is a protein that can bind to LRP-5/6 to block Wnt signaling.

Osteosarcoma patient revealed LRP-5 expression at a very high rate $(50 \%)$ in tumor samples, and the presence of LRP-5 correlated with tumor metastasis and the chondroblastic subtype of osteosarcoma.

Mesenchymal/stromal cells (MSC) have the potential to differentiate into osteoblasts in certain culture conditions and are candidates for therapeutic applications. MSC are consistently expressed Wnt signal components, such as LRP-5, Dkk-1, sFRPs, GSK-3b, and TCFs. ${ }^{50}$ After treatment with Wnt3a or Litium, cells promote canonical Wnt signaling by inhibiting GSK-3b, reduce phosphorylated $\beta$-catenin in cytosol, and increase $\beta$-catenin nuclear translocation. Thus, autocrine Wnt signaling operates in primitive MSC populations and regulates self-renewal and lineage-specific differentiation. A similar line of experiments using osteo-progenitors calvarial cells,${ }^{51}$ which expresses collagen $2.3 \mathrm{~kb}$-driven GFP protein, exhibited higher expression of Wnt3a, LRP-5, and TCF1 in addition to BMPs, BMP antagonists, and IGF-1. After a long-term 
culture under osteogenic factors, the GFP positive cells showed a higher expression in some BMP antagonist, BAMBI (BMP and activin membrane bound inhibitors), as compared to that in GFP negative cells. The expression levels of BMPs and other BMP antagonists, such as Cerberus, follistatin, and TSG protein were not changed.

$\beta$-Catenin-targeted null mice show embryonic lethality with a lack of skeletal structures derived from cranial neural crest. ${ }^{52}$ Some mutation in Wnt signaling can produce a bone phenotype without growth retardation. Loss-of-function mutation in LRP-5 results in low bone mass in humans and mice. ${ }^{53}$ Conversely, a null mutation of the Wnt antagonist sFRPs results in a high bone mass. ${ }^{54}$ These data suggest that wnt signaling stimulates osteoblast differentiation and thereby increases bone formation. However, increased bone mass is not only the result of increased bone formation but also of decreased bone resorption. A recent study by the Karsenty group using a stable form of $\beta$-catenin and colIa promotor-driven $\beta$-catenin deletion demonstrated that $\mathrm{Wnt}$ signals do not affect bone formation but affect bone resorption by elevating osteoprotegerin expression in osteoblasts. ${ }^{55}$ They could not observe any changes in the expression of osteoblast differentiation markers. This result raised the question whether Wnt signals positively affect osteoblast differentiation as proposed from the results obtained from transgenic mice and null mice of LRP. $\beta$-catenin deletion in limb and head mesenchyme showed that Wnt signals determine an early stage of mesenchymal cell differentiation to osteoblasts. ${ }^{56}$ If Wnt signals are not sufficient, cells prefer to differentiate into chondrocytes rather than osteoblasts. Taken together, these results suggest Wnt signals might intensively affect mesenchymal lineage cell differentiation.

Both BMP and Wnt signaling promote osteogenesis. How do they interact in the signaling process? Wnt signaling promotes osteogenesis by directly stimulating RUNX2 (cbfa-1) gene expression ${ }^{57}$ and this process is blocked by sFRP-1. In fact, sFRP-1 null mice displayed high bone mass with higher expression of RUNX2, TCF1, and osteocalcin in bone samples. ${ }^{54}$ Interestingly, Wnt signaling similar to BMP signaling directly regulates RUNX2 transcriptional activity. In fact, an RUNX2 promotor retains a TCF consensus sequence with proximity to a RUNX2 regulatory site. Cooperative signaling pathways of BMP and Wnt are proved by the use of Wnt signal inhibitors. ${ }^{58}$ The ability of BMP2 to induce alkaline phophatase was inhibited by blocking Wnt/LRP-5 signals in mesenchymal cells, and conversely, sclerostin and noggin inhibit Wnt-induced osteoblast differentiation. An independent mechanism between BMPs and Wnt signaling has been shown by others. ${ }^{59} \mathrm{~A} \beta$-catenin mutant with constitutive transcriptional activity (deltaN151) did not stimulate osteogenic differentiation in multipotent embryonic cells $\mathrm{C} 3 \mathrm{H} 10 \mathrm{~T} 1 / 2$ by itself; however, in the presence of BMP-2 osteoblast marker expression along with matrix mineralization was markedly enhanced. Similarly, the active mutants of the $\beta$-catenin and BMP-2 synergistically stimulated new bone formation in mouse calvaria, and also the synergism was explained by a RUNX2-independent mechanism. 
Thus, it may be suggested that BMP and Wnt signals use a common pathway at some points and depend on cells and systems used.

Close interplay between the Wnt signal and BMP signals has been reported. ${ }^{60}$ Noggin interacts with Dkk1 in head induction, and BMPs regulate the expression level of Dkk1 in limb development. These interactions may explain that the binding sites of BMP antagonists to Wnt protein are different from the site bound to BMPs.

\section{SUMMARY}

We believe that current studies have provided some evidence that BMPs and BMP antagonists play an important role in adult bone remodeling. To study the interaction mechanism of BMP and Wnt signaling may give rise to new therapeutic means for osteoporosis.

\section{ACKNOWLEDGMENT}

We thank Dr. Zaidi for reading this manuscript and making the discussion focused for writing.

\section{REFERENCES}

1. WozNEY, J.M. 1993. Bone morphogenetic proteins and their gene expression. In Cellular and Molecular Biology of Bone. M. Noda, Ed.: 131-167. Academic Press. San Diego, CA.

2. BotchKarev, V. \& A.A. Sharov. 2004. BMP signaling in the control of skin development and hair follicle growth. Differentiation 72: 512-526.

3. NeEDHAM, S.J., M. BRITTAN, S.A. MCDonald, et al. 2005. Intestinal stem cells. J. Cell Mol. Med. 9: 11-24.

4. Kishigami, S. \& Y. Mishina. 2005. BMP signaling and early embryonic patterning. Cytokine Growth Factor Rev. 16: 265-278.

5. Tsumaki, N. \& H. YoshiKaWa. 2005. The role of bone morphogenetic proteins in endochondral bone formation. Cytokine Growth Factor Rev. 16: 279-285.

6. KugimiYa, F., H. Kawaguchi, S. KameKURA, et al. 2005. Involvement of endogenous bone morphogenetic protein (BMP) 2 and BMP6 in bone formation. J. Biol. Chem. 280: 35704-35712.

7. GaZzerRo, E., V. GANGJI \& E. CANALIs. 1998. Bone morphogenetic proteins induce the expression of noggin, which limits their activity in cultured rat osteoblasts. J. Clin. Invest. 10: 2106-2114.

8. Aвe E, M. Yamamoto, Y. TAGUChI, et al. 2000. Essential requirement of BMPs$2 / 4$ for both osteoblast and osteoclast formation in murine bone marrow cultures from adult mice: antagonism by noggin. J. Bone Miner. Res. 15: 663-673.

9. Moon, R.T., A.D., Kohn, G.V. DeFerrari, et al. 2004. Wnt and $\beta$-catenin signaling: diseases and therapies. Nature Rev. Genet. 5: 689-699. 
10. Miyazono, K., S. Maeda \& T. Imamura. 2005. BMP receptor signaling: transcriptional targets, regulation of signals and signaling cross-talk. Cytokine Growth Factor Rev. 16: 251-263.

11. Nohe, A., E. Keating, P. Knaus, et al. 2004. Signal transduction of bone morphogenetic protein receptors. Cell. Signal. 16: 291-299.

12. Chen, D.I., M. Zhao \& G.R. Mundy. 2004. Bone morphogenetic proteins. Growth Factors 22: 233-241.

13. Hullinger, T.G., Q. Pan, H. Viswanathan, et al. 2001. TGFbeta and BMP2 activation of the OPN promoter: roles of smad-and hox-binding elements. Exp. Cell Res. 262: 69-74.

14. WAN, M., X. ShI, X. Feng, et al. 2001. Stimulation of osteoprotegerin (OPG) gene expression by transforming growth factor-beta (TGF-beta). Mapping of the OPG promoter region that mediates TGF-beta effects. J. Biol. Chem. 276: 36241-36250.

15. Stopa, M., D. Anhuf, L. Trestegen, et al. 2000. Participation of smad2, smad3, and smad4 in transforming growth factor beta (TGF-beta) induced activation of smad7. J. Biol. Chem. 275: 29308-29317.

16. Guicheux, J., J. Lemonnier, C. GHAYOR, et al. 2003. Activation of p38 mitogenactivated protein kinase and c-Jun-NH2-terminal kinase by BMP-2 and their implication in the stimulation of osteoblastic cell differentiation. J. Bone Miner. Res. 18: 2060-2068.

17. OsyczKa, A.M. \& P.S. LeBoy. 2005. Bone morphogenetic protein regulation of early osteoblast genes in human marrow stromal cells is mediated by extracellular signal-regulated kinase and phosphatidylinositol 3-kinase signaling. Endocrinology 146: 3428-3437.

18. Zimmerman, L.B., J.M. De Jesus-Escobar \& R.M. Harland. 1996. The Spemann organizer signal, noggin, binds and inactivates bone morphogenetic protein 4 . Cell 86: 599-606.

19. LARRAIN, J., D. BACHILler, B. Lu, et al. 2000. BMP-binding modules in chordin: a model for signalling regulation in the extracellular space. Development 127: 821-830.

20. Canalis, E., A. Economides \& E. Gazzerro. 2003. Bone morphogenetic proteins, their antagonists, and the skeleton. Endocr. Rev. 24: 218-235.

21. Yanagita, M. 2005. BMP antagonists: their roles in development and involvement in pathophysiology. Cytokine Growth Factor Rev. 16: 309-317.

22. Kuriyama, S., G. LuPo, K. OHTA, et al. 2006. Tsukushi controls ectodermal patterning and neural crest specification in Xenopus by direct regulation of BMP4 and X-delta-1 activity. Development 133: 75-88.

23. Brunet, L.J., J.A. MCMAhon, A.P. MCMAhon, et al. 1998. Noggin, cartilage morphogenesis, and joint formation in the mammalian skeleton. Science 280: 1455-1457.

24. Gong, Y., D. Krakow, J. Marcelino, et al. 1999. Heterozygous mutations in the gene encoding noggin affect human joint morphogenesis. Nat. Genet. 21: 302-304.

25. Gazzerro, E., V. Gangu \& E. Canalis. 1998. Bone morphogenetic proteins induce the expression of noggin, which limits their activity in cultured rat osteoblasts. J. Clin. Invest. 10: 2106-2114.

26. Wu, X.B., Y. LI, A. SCHNEIDER, et al. 2003. Impaired osteoblastic differentiation, reduced bone formation, and severe osteoporosis in noggin-overexpressing mice. J. Clin. Invest. 112: 924-934. 
27. NaKase, T., S. Nomura, H. Yoshikawa, et al. 1994. Transient and localized expression of bone morphogenetic protein 4 messenger RNA during fracture healing. J. Bone Miner. Res. 9: 651-659.

28. Kloen, P., M. Di Paola, O. Borens, et al. 2003. BMP signaling components are expressed in human fracture callus. Bone 33: 362-371.

29. Devlin, R.D., Z. Du, R.C. Pereira, et al. 2003. Skeletal overexpression of noggin results in osteopenia and reduced bone formation. Endocrinology 144: 19721978.

30. Bachiller, D., J. Klingensmith, N. Shneyder, et al. 2003. The role of chordin/Bmp signals in mammalian pharyngeal development and DiGeorge syndrome. Development 130: 3567-3578.

31. Zhang, D., C.M. Ferguson, R.J. O'Keefe, et al. 2002. A role for the BMP antagonist chordin in endochondral ossification. J. Bone Miner. Res. 17: 293300.

32. Anderson, R.M., A.R. Lawrence, R.W. Stottmann, et al. 2002. Chordin and noggin promote organizing centers of forebrain development in the mouse. Development 129: 4975-4987.

33. Gazzerro, E., V. Deregowski, S. Vaira, et al. 2005. Overexpression of twisted gastrulation inhibits bone morphogenetic protein action and prevents osteoblast cell differentiation in vitro. Endocrinology 146: 3875-3882.

34. Nosaka. T., S. Morita, H. Kitamura, et al. 2003. Mammalian twisted gastrulation is essential for skeleto-lymphogenesis. Mol. Cell Biol. 23: 2969-2980.

35. Staehling-Hampton, K., S. Proll, B.W. Paeper, et al. 2002. A 52-kb deletion in the SOST-MEOX1 intergenic region on 17q12-q21 is associated with van Buchem disease in the Dutch population. Am. J. Med. Genet. 110: 144-152.

36. Kasu, N., J. LAurikKala, M. Imanishi, et al. 2003. Sclerostin is a novel secreted osteoclast-derived bone morphogenetic protein with unique ligand specificity. J. Biol. Chem. 278: 24113-24117.

37. Winkler, D., M.K. Sutherl, J. Geoghegan, et al. 2003. Osteocyte control of bone formation via sclerostin, a novel BMP antagonist. EMBO J. 22: 62676276.

38. VAn Bezooijen, R.L., B.A.J. Roelen, A. Visser, et al. 2004. Sclerostin is an osteocyte negative regulator of bone formation, but not a classical BMP antagonist. J. Exp. Med. 199: 805-814.

39. LI, X., Y. ZHANG, H. KANG, et al. 2005. Sclerostin binds to LRP5/6 and antagonizes canonical Wnt signaling. J. Biol. Chem. 280: 19883-19887.

40. JiLKA, R.L., R.S. WeInStein, K. TAKAhAShi, et al. 1996. Linkage of decreased bone mass with impaired osteoblastogenesis in a murine model of accelerated senescence. J. Clin. Invest. 97: 1732-1740.

41. Silva, M.J., M.D. Brodt \& B.A. Uthgenannt. 2004. Morphological and mechanical properties of caudal vertebrae in the SAMP6 mouse model of senile osteoporosis. Bone 35: 425-431.

42. Kaplan, F.S. \& E.M. Shore. 2000. Progressive osseous heteroplasia. J Bone Miner Res. 15: 2084-2094.

43. KAN L., M. Hu, WA. Gomes, et al. 2004. Transgenic mice overexpressing BMP4 develop a fibrodysplasia ossificans progressiva (FOP)-like phenotype. Am. J. Pathol. 165: 1107-1115.

44. ZHAO, M., S.E. HARRIS, D. Horn, et al. 2002. Bone morphogenetic protein receptor signaling is necessary for normal murine postnatal bone formation. J. Cell Biol. 157: $1049-1060$. 
45. Yamashita, M., Y. SAI-XiA, Z. Gen-MU, et al. 2005. Ubiquitin ligase smurf1 control osteoblast activity and bone homeostases by targeting MEKK2 for degradation. Cell 121: 101-113.

46. Mishina, Y., M.W. Starbuck, M.A. Gentile, et al. 2004. Bone morphogenetic protein 1A receptor signaling regulates postnatal osteoblast function and bone remodeling. J. Biol. Chem. 279: 27560-27566.

47. LIU, Z., W. SHI, X. Ji, et al. 2004. Molecules mimicking smad1 interacting with Hox stimulate bone formation. J. Biol. Chem. 279: 11313-11319.

48. Oshima, T., M. ABE, J. AsAno, et al. 2005. Myeloma cells suppress bone formation by secreting a soluble Wnt inhibitor, sFRP-2. Blood. 106: 3160-3165.

49. Hoang, B.H., T. Kubo, J.H. Healey, et al. 2004. Expression of LDL receptorrelated protein 5 (LRP5) as a novel marker for disease progression in high-grade osteosarcoma. Int. J. Cancer 109: 106-111.

50. Etheridge, S.L., G.J. Spencer, D.J. Heath, et al. 2004. Expression profiling and functional analysis of wnt signaling mechanisms in mesenchymal stem cells. Stem Cells 22: 849-860.

51. Bilic-Curcic, I., M. Kronenberg, X. Jiang, et al. 2005. Visualizing levels of osteoblast differentiation by a two-color promoter-GFP strategy: type I collagenGFPcyan and osteocalcin-GFPtpz. Genesis 43: 87-98.

52. Haegel, H., L. LARUe, M. OHSUGI, et al.1995. Lack of beta-catenin affects mouse development at gastrulation. Development 121: 3529-3537.

53. Kato, M., M.S. Patel, R. LeVasseur, et al. 2002. Cbfa1-independent decrease in osteoblast proliferation, osteopenia, and persistent embryonic eye vascularization in mice deficient in Lrp5, a Wnt coreceptor. J. Cell Biol. 157: 303-314.

54. Bodine, P.V., W. ZhaO, Y.P. Kharode, et al. 2004. The Wnt antagonist secreted frizzled-related protein-1 is a negative regulator of trabecular bone formation in adult mice. Mol. Endocrinol. 18: 1222-1237.

55. Glass, D.A. II, P. BialeK, J.D. Ahn, et al. 2005. Canonical Wnt signaling in differentiated osteoblasts controls osteoclast differentiation. Dev. Cell 8: 751764.

56. Hill, T.P., D. Spater, M.M. TAKeto, et al. 2005. Canonical Wnt/beta-catenin signaling prevents osteoblasts from differentiating into chondrocytes. Dev. Cell 8: 727-738.

57. Gaur, T., C.J. Lengner, H. Hovhannisyan, et al. 2005. Canonical WNT signaling promotes osteogenesis by directly stimulating Runx 2 gene expression. J. Biol. Chem. 280: 33132-33140.

58. RAwAdi, G., B. VAyssiere, F. DunN, et al. 2003. BMP-2 controls alkaline phosphatase expression and osteoblast mineralization by a Wnt autocrine loop. J. Bone Miner. Res. 18: 1842-1853.

59. Mbalaviele, G., S. Sheikh, J.P. Stains, et al. 2005. Beta-catenin and BMP-2 synergize to promote osteoblast differentiation and new bone formation. J. Cell. Biochem. 94: 403-418.

60. Mukhopadhyay, M., S. Shtrom, C. Rodriguez-Esteban, et al. 2001. Dickkopf1 is required for embryonic head induction and limb morphogenesis in the mouse. Dev. Cell 1: 423-434. 